\title{
Extending the depth of human plasma proteome coverage using simple fractionation techniques
}

\author{
Gurjeet Kaur¹, Anne Poljak ${ }^{1,2 *}$, Syed Azmal Ali ${ }^{4}$, Ling Zhong², Mark Raftery², Perminder \\ Sachdev ${ }^{1,3}$
}

${ }^{1}$ Centre for Healthy Brain Ageing, School of Psychiatry, University of New South Wales, Sydney, NSW, 2052, Australia

${ }^{2}$ Mark Wainwright Analytical Centre, Bioanalytical Mass Spectrometry Facility, University of New South Wales, Sydney, NSW, 2052, Australia

${ }^{3}$ Neuropsychiatric Institute, Euroa Centre, Prince of Wales Hospital, Sydney, NSW, 2052, Australia

${ }^{4}$ Cell biology and proteomics lab, National Dairy Research Institute, Karnal, Haryana, 132001, India

\section{Supplementary Figures}

Supplementary Figure 1. Representative NuPAGE LDS gel profile of undepleted plasma and depleted plasma containing low abundant plasma proteins (LAP) and high abundant plasma proteins (HAP) depleted of high abundance proteins using HU6, HU14 and ProteoMiner depletion methods. Each gel lane contained and equal loading of total protein $(50 \mu \mathrm{g}$ total proteins were loaded per gel lane).

Supplementary Figure 2. Multiscatter plot depicting the cross correlation of all the methods (120 correlations in total). The LFQ intensities of each of the 16 individual sample types down to technical replicate level are shown on either axis, scatter plots are shown in orange and Pearson correlation values are shown below each scatter plot.

Supplementary Figure 3. Venn diagram showing overlap of top 100 dynamic range of protein between depletion methods A. HU6 C18 and HU6 SDS PAGE, B. HU14 C18 and HU14 SDS PAGE, C. PB C18 and PB SDS PAGE D. PUB C18 and PUB SDS PAGE. 


\section{Supplementary Tables}

Supplementary Table 1. The full table describing the identification of the analysed MaxQuant results of HU6-SDS PAGE and C18.

Supplementary Table 2. The full table describing the identification of the analysed MaxQuant results of HU14-SDS PAGE and C18.

Supplementary Table 3. The full table describing the identification of the analysed MaxQuant results of PB-SDS PAGE and C18.

Supplementary Table 4. The full table describing the identification of the analysed MaxQuant results of PUB-SDS PAGE and C18.

Supplementary Table 5.The full list of protein identifications using the Trans Proteomics Pipeline (TPP), and resulting from use of three search engines (Mascot, Comet and XTandem), and combining all 8 sample types (HU6-SDS PAGE and C18, HU14-SDS PAGE and C18, PB-SDS PAGE and C18, PUB- SDS PAGE and C18), consisting of 24 fractions and 2 technical replicates and resulting in 384 raw files.

Supplementary Table 6: A. MaxQuant parameter set and B. Parameter set for TPP, which were used for processing all the raw files generated by LCMSMS of the samples.

Supplementary Table 7: MaxQuant derived information on the raw peptide matches for each of the 4 high abundance protein removal steps.

Supplementary Table 8. Detailed breakdown of the protein numbers mapped to 51 specific organs separately from the Human Protein Atlas (HPA) (https://www.proteinatlas.org/). 


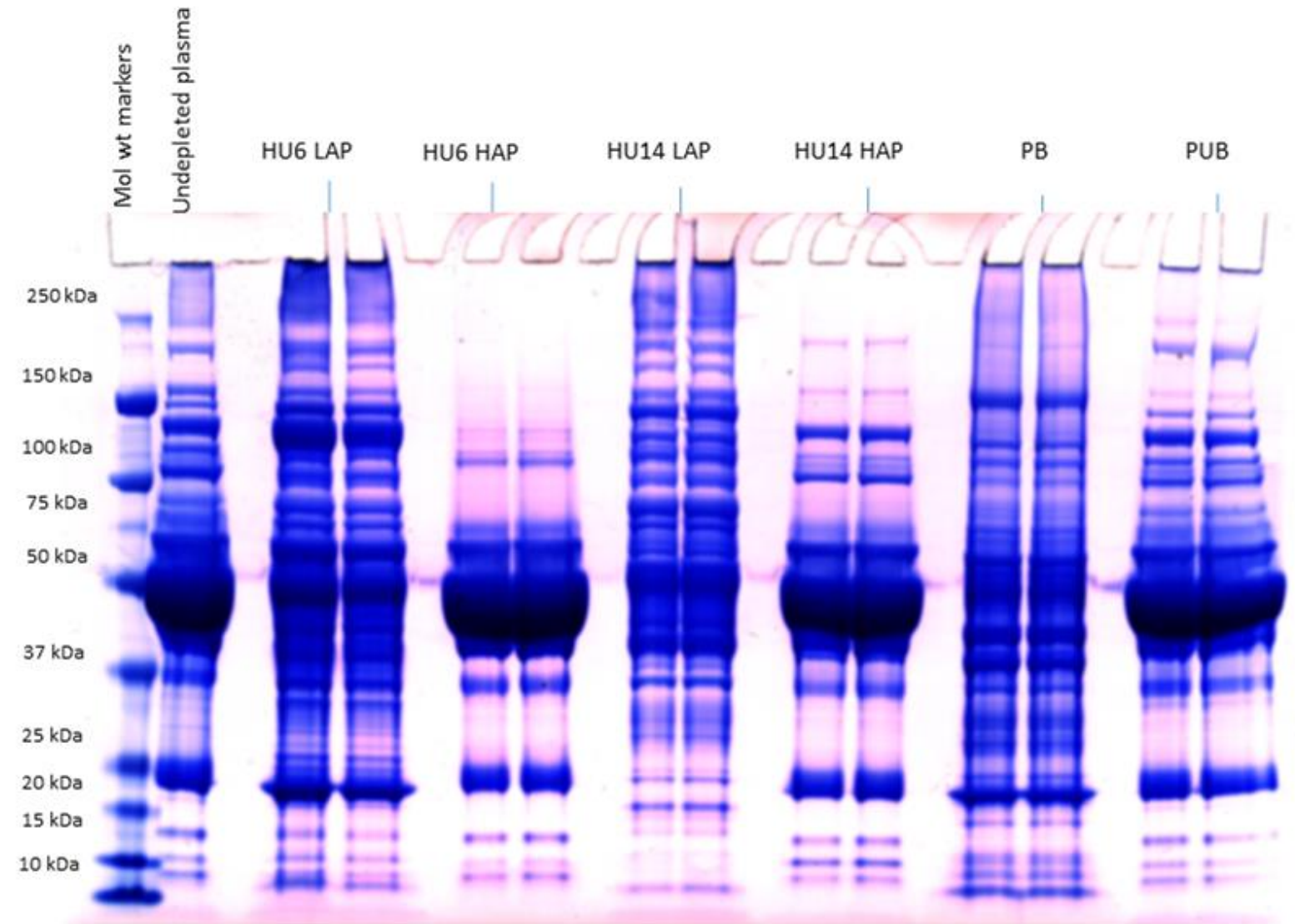

Supplementary Figure 1. Representative NuPAGE LDS gel profile of undepleted plasma and depleted plasma containing low abundant plasma proteins (LAP) and high abundant plasma proteins (HAP) depleted of high abundance proteins using HU6, HU14 and ProteoMiner depletion methods. Each gel lane contained and equal loading of total protein $(50 \mu \mathrm{g}$ total proteins were loaded per gel lane). 


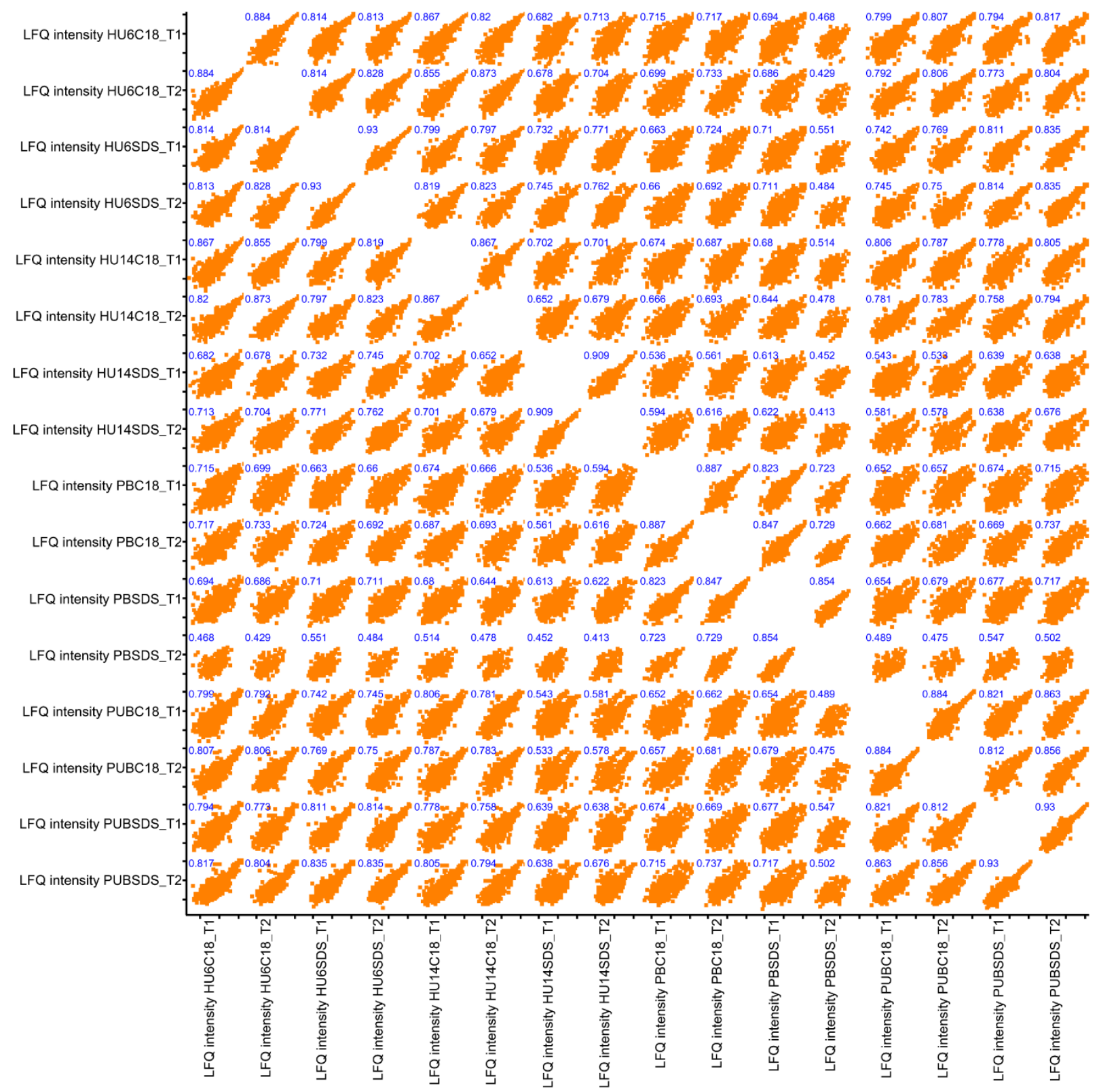

Supplementary Figure 2. Multiscatter plot depicting the cross correlation of all the methods (120 correlations in total). The LFQ intensities of each of the 16 individual sample types down to technical replicate level are shown on either axis, scatter plots are shown in orange and Pearson correlation values are shown below each scatter plot. 
A

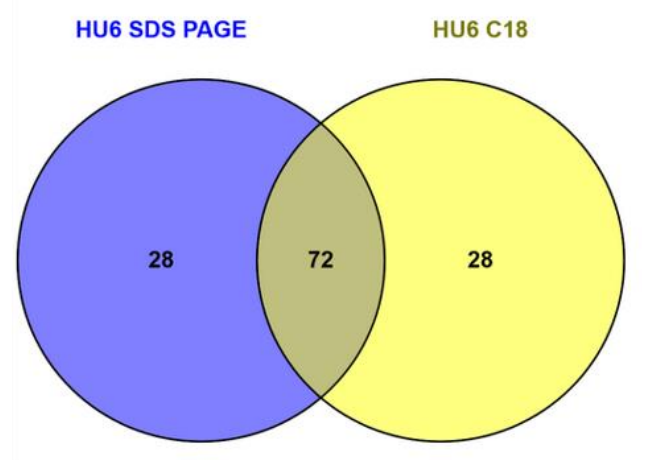

$\mathrm{C}$

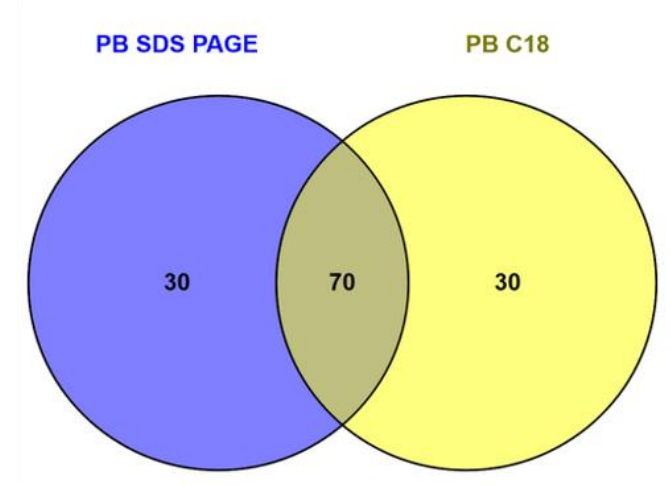

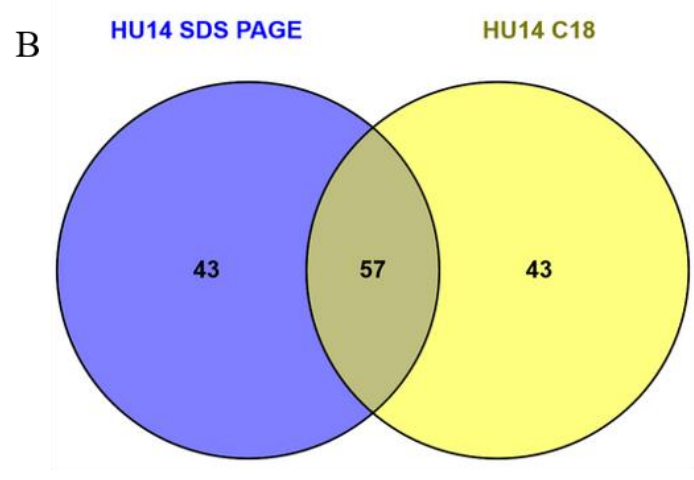

$\mathrm{D}$

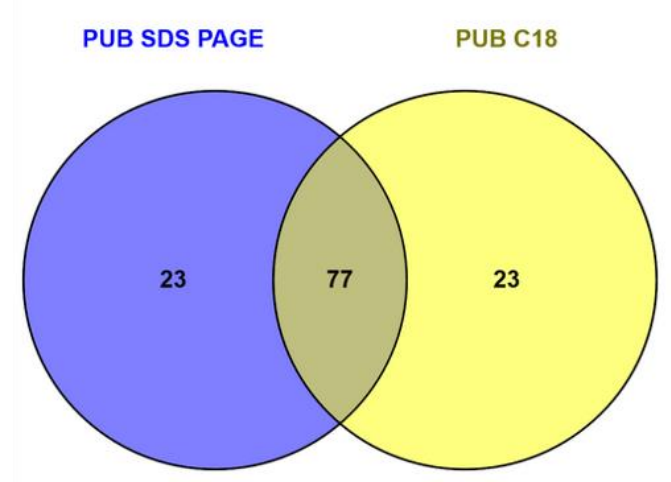

Supplementary Figure 3. Venn diagram showing overlap of top 100 dynamic range of protein between depletion methods A. HU6 C18 and HU6 SDS PAGE, B. HU14 C18 and HU14 SDS PAGE, C. PB C18 and PB SDS PAGE D. PUB C18 and PUB SDS PAGE. 